\title{
Glucosamine Activates $T$ Lymphocytes in Healthy Individuals and may Induce GVHD/GVL in Stem Cell Transplanted Recipients
}

\author{
Behnam Sadeghi ${ }^{1}$, Hans Hägglund ${ }^{2}$, Mats Remberger ${ }^{3}$, Sulaiman Al-Hashmi ${ }^{1}$, Zuzana Hassan ${ }^{1,3}$, \\ Manuchehr Abedi-Valugerdi ${ }^{4}$ and Moustapha Hassan ${ }^{*}, 1,5$
}

\author{
${ }^{1}$ Experimental Cancer Medicine, Institution for Laboratory Medicine, Karolinska Institutet, Stockholm, Sweden \\ ${ }^{2}$ Karolinska Haematology Centre, Department of Medicine, Karolinska University Hospital, Huddinge, and Karolinska \\ Institutet, Stockholm, Sweden \\ ${ }^{3}$ Center for Allogeneic Stem Cell Transplantation (CAST), Karolinska University Hospital-Huddinge, Stockholm, \\ Sweden \\ ${ }^{4}$ Department of Biochemistry and Biophysics, Arrhenius Laboratories for the Natural Sciences, Stockholm University, \\ Stockholm, Sweden \\ ${ }^{5}$ Clinical Research Centre, KFC, Novum, Karolinska University Hospital-Huddinge, Stockholm, Sweden
}

\begin{abstract}
Graft versus host disease (GVHD) is the major limiting factor after Hematopoietic stem cell transplantation (HSCT). In this study, we report a HSCT-patient who developed arthritis 10-month after allogeneic sibling transplantation, treated with glucosamine-hydrochloride and developed severe acute GVHD within three weeks after the administration of glucosamine. Another HSCT-recipient with an increase in CD33+cells received one dose of donor lymphocyte infusion (DLI). Due to the lack of DLI and based on our experience from the first case, the patient was treated with glucosamine. No sign of relapse was observed in the second patient despite increased number of CD33+ for more than three years. The glucosamine effect was evaluated in seven individuals given the drug for four weeks. The effect of glucosamine treatment was examined by mixed-lymphocyte-reaction (MLR) and the levels of soluble IL-2 receptor (sIL$2 \mathrm{R}), \mathrm{TNF}-\alpha$ and IFN- $\gamma$ were determined. Glucosamine administration exhibited an enhancement in the allogeneic MLR and an increase in the serum levels of sIL-2R, but a decrease in the serum levels of inflammatory cytokines TNF- $\alpha$ and IFN- $\gamma$.

We conclude that glucosamine may act as an activator of the immune system. In HSCT-patients, glucosamine-mediated immune activation might result in either beneficial or deleterious outcomes. Thus, precautions should be taken when glucosamine is prescribed to HSCT-patients.
\end{abstract}

Keywords: Glucosamine, GVHD, GVL, Stem cell transplantation, AML.

\section{INTRODUCTION}

Allogeneic hematopoietic stem cell transplantation (HSCT) is curative treatment for patients suffering from haematological malignancies such as leukaemia and lymphoma [1] and non haematological malignancies e.g. solid tumours. Moreover, HSCT is the first hand choice for the treatment of several genetic disorders and inborn errors of metabolism. Despite the success of HSCT still transplantation related complications such as graft versus host disease (GVHD), veno-occlusive disease (VOD) and treatment related toxicity are the major limiting factors.

The allograft usually contains $\mathrm{T}$ lymphocytes that can prevent graft rejection and mediate a graft-versus-leukemia (GVL) effect, which is beneficial for the patients [2]. However, donor T lymphocytes can also initiate GVHD, that jeopardize the transplantation outcome and may increase

*Address correspondence to this author at the ECM, KFC, Novum, Karolinska University Hospital-Huddinge, S-141 86 Stockholm, Sweden; Tel: +46-8-58583862; E-mail: moustapha.hassan@ki.se both morbidity and mortality [3]. The development of GVHD depends on variety of factors including pretransplant conditioning, immunosuppressive treatment and tissue damage through activation of donor allo-reactive $\mathrm{T}$ lymphocytes against host organs [3]. Although, the underlying mechanisms for the development of GVHD and GVL effects are not fully understood, in vivo activation of donor $\mathrm{T}$ lymphocytes has been considered as the primary event for the initiation of these processes $[2,3]$. Thus, it is conceivable that the employed pre- and post-transplant medications that exert immunosuppressive or immunostimulatory properties might have profound effects on the outcome of HSCT.

Glucosamine is a form of amino sugar that is synthesized by virtually all cells in the human body and has various physiological activities including the stimulation of the synthesis of proteoglycans, inhibition of the degradation of proteoglycans and the inhibition of the synthesis of proteolytic enzymes and other substances that contribute to damage of cartilage matrix [4-6]. Owing to its beneficial effects on the reconstitution of joint cartilage, glucosamine has been clinically used as a supplementary drug to treat 
osteoarthritis (OA) [7]. At present, glucosamine is sold as an over-the-counter (OTC) drug in many countries. However, recently Wilkens et al. have reported in a large clinical study that glucosamine did not result in reduced pain-related disability after the 6-month intervention and after 1-year follow-up in chronic low back pain (LBP) with degenerative lumbar osteoarthritis (OA) patients [8].

In addition to its physiological properties, glucosamine is suggested to possess anti-inflammatory and immunsuppressive effects. For instance, several studies have shown that this substance inhibits the synthesis of proinflammatory mediators in human chondrocytes in vitro [9], suppresses the activation of NF- $\kappa B$ and modulates the bioactivity of IL- $1 \beta$ in rat chondrocytes [10], and suppresses unprimed T-cell responses by interfering with antigen-presenting cell functions and by a direct inhibitory effect on anti-CD3-induced proliferation of murine $\mathrm{T}$ cells [11]. However, experimental studies suggest that glucosamine functions as an immunomodulator rather than as an immunosuppressant in vivo, i.e., systemic administration of this compound is shown to abrogate the acute phase of murine autoimmune encephalomyelitis by redirecting the disease mediated Thelper 1 (Th1) type of response to a more protective Th2 response [12]. Thus, it is conceivable that depending on the type of the disease, the use of glucosamine can cause either beneficial or deleterious immune mediated effects.

In the present study, we report that treatment with glucosamine activates the immune system in such a way that may lead to the development of acute graft-versus-host disease and/or graft versus leukaemia effect in patients transplanted with allogeneic stem cells. Furthermore, we were able to show that healthy volunteers treated with glucosamine exhibit an enhancement in the allogeneic mixed lymphocyte reaction and an increase in the serum levels of soluble IL-2 receptor (sIL-2R), but a decrease in the serum levels of inflammatory cytokines including TNF- $\alpha$ and IFN- $\gamma$.

\section{PATIENTS AND METHODS}

\section{a) Case 1}

A 63-year-old male with acute myeloid leukaemia (AML, M2) and severe myelofibrosis secondary to polycythaemia vera underwent haematopoietic stem cell transplantation with mobilized peripheral blood stem cells (PBSC) from an HLA (A, B- and DR) -identical sibling donor after reduced intensity conditioning with fludarabine, $30 \mathrm{mg} / \mathrm{m}^{2}$ daily for 5 days, and treosulfan, $14 \mathrm{~g} / \mathrm{m}^{2}$ for three consecutive days. Patient received PBSC as stem cell-source at the dose of $3.6 \times 10^{6} / \mathrm{kg} \mathrm{CD} 34$ and $135 \times 10^{6} / \mathrm{kg}$ CD3+. Prophylaxis for graft-versus-host disease consisted of cyclosporine $(1 \mathrm{mg} / \mathrm{kg}$, iv) at day -1 and 0 , followed by a dose of $3 \mathrm{mg} / \mathrm{kg}$ (po). Cyclosporine was given in combination with four doses of methotrexate $\left(15 \mathrm{mg} / \mathrm{m}^{2}\right.$ at day +1 and $10 \mathrm{mg} / \mathrm{m}^{2}$ at days $+3,+6$ and +11$)$. Three months after transplantation the immunosuppressive treatment was discontinued and there were no signs of GVHD. Patient developed full donor chimerism $(100 \%)$ in $\mathrm{T}-$, B- and myeloid cell lineages in the bone marrow and he was in complete remission. Ten months after transplantation, the patient was treated with a dose of $625 \mathrm{mg}$ twice daily of
Artrox $^{\circledR} \quad$ (Glucosamine hydrochloride) for relieving the symptoms of his osteoarthritis.

\section{b) Case 2}

A 64-year-old female with acute myeloid leukaemia (AML, M1) in second complete remission (CR2) received a reduced intensity conditioning followed by mobilized peripheral blood stem cells (PBSC) from a 71 years old HLA-A, B- and DR -identical sibling. The preparative regimen consisted of fludarabine $\left(30 \mathrm{mg} / \mathrm{m}^{2} /\right.$ day for 6 days $)$, busulfan $(4 \mathrm{mg} / \mathrm{kg} /$ day for 2 days) and ATG $(2 \mathrm{mg} /$ day for 4 days). Patient received PBSC as stem cell source at the dose of $14.6 \times 10^{6} / \mathrm{kg} \mathrm{CD} 34+$ and $65 \times 10^{6} / \mathrm{kg}$ CD3+. Immunosuppressive treatments for prevention of graft-versus-host disease were cyclosporine as an iv dose $1 \mathrm{mg} / \mathrm{kg}$, at day -1 and 0 , followed by an oral dose of $3 \mathrm{mg} / \mathrm{kg}$ given in combination with four doses of methotrexate $\left(15 \mathrm{mg} / \mathrm{m}^{2}\right.$ at day +1 and $10 \mathrm{mg} / \mathrm{m}^{2}$ at days $+3,+6$ and +11$)$. Both drugs were discontinued 4 months after transplantation. The patient did not develop acute or chronic GVHD.

The patient was in CR with a stable mixed chimerism (90-95\% donor T-cells and 100\% donor myeloid cells) in the bone marrow 12 months after HSCT. Due to increased recipient $\mathrm{T}(>40 \%)$ and myeloid cells $(7 \%)$ in the peripheral blood, twenty months after HSCT, the patient received just one donor lymphocyte infusion (DLI) at a dose of $0.43 \times 10^{6}$ $\mathrm{CD} 3+$ cells $/ \mathrm{kg}$. Due to the general poor health status of the donor, no more lymphocytes could be harvested and hence DLI could be repeated. Therefore, and based on the experience seen in case 1, Arthrox ${ }^{\circledR}$ (Glucosamine hydrochloride) at a dose of $625 \mathrm{mg}$ twice daily was started one month after DLI (21 months post transplantation).

\section{c) Control Group}

Eight AML patients were considered as control group. Mean age was 48.5 years (35-59). Conditioning consist of busulfan-cyclophosphamide (Bu-Cy; 3 patients), fludarabine-busulfan (Flu-Bu; 4 patients) and treoulfan-Flu (one patient) followed by stem cell transplantation using $9.64 \mathrm{x}$ $10^{6} / \mathrm{kg}$ for CD34+ and $294 \times 10^{6} / \mathrm{kg}$ for CD3+. Preventive treatment for GVHD was cyclosporine as an i.v. dose 1 $\mathrm{mg} / \mathrm{kg}$, at day -1 and 0 , followed by an oral dose of $3 \mathrm{mg} / \mathrm{kg}$ in combination with four doses of methotrexate $\left(15 \mathrm{mg} / \mathrm{m}^{2}\right.$ at day +1 and $10 \mathrm{mg} / \mathrm{m}^{2}$ at days $+3,+6$ and +11 ) for the patients.

\section{d) Treatment with Glucosamine in Healthy Volunteers}

In order to evaluate the effects of glucosamine treatment on the immune system, seven healthy individuals, 4 males and 3 females, with a median age of 49 (range 28-63) years were treated with glucosamine (625 mg twice daily) for 4 weeks. None of the volunteers had any history of infectious disease for at least four weeks before and during the treatment with glucosamine. The study protocol was approved by the Ethical Committee at the Karolinska University Hospital.

\section{MIXED LYMPHOCYTE REACTION}

Blood samples were taken before, 2, 4 and 12 weeks after glucosamine administration. Lymphocytes were isolated on a 
Ficoll-Hypaque gradient and resuspended in RPMI 1640, supplemented with $10 \%$ human $\mathrm{AB}$ serum, at a concentration of $10^{6} \mathrm{cells} / \mathrm{mL}$. A pool of 7 individuals were irradiated (20 Gy) and used as stimulator cells. Stimulator and responder cells $\left(100 \mu \mathrm{L}\right.$ of each) were cultured at $37^{\circ} \mathrm{C}$ and $5 \% \mathrm{CO}_{2}$ on micro titre plates for 5 days. $1 \mu \mathrm{Ci}$ of ${ }^{3} \mathrm{H}$ thymidine was added $24 \mathrm{~h}$ prior to harvest.

\section{Cytokine Measurement}

Samples were allowed to clot and serums were frozen and stored at $-20^{\circ} \mathrm{C}$ until analyzed. The levels sIL-2R, TNF $\alpha$ and IFN- $\gamma$ in samples were determined employing automated chemo-luminescence immunoassay (IMMULITE ${ }^{\circledR}$, Siemens Healthcare Diagnostics Inc., IL, USA).

\section{Statistical Analysis}

All data are expressed as means \pm SD (standard deviation) unless otherwise mentioned. Differences between groups were analyzed using Mann-Whitney (U-test). $\mathrm{P}<0.05$ is considered statistically significant unless otherwise stated. All statistical analyses were performed utilizing Statistica software (ver. 8).

\section{RESULTS}

\section{Case 1}

Three weeks after glucosamine administration, the patient developed diarrhoea ( $>15$ times/day) and abdominal pain. Histopathological examination of a colon biopsy specimen revealed acute GVHD grade III. The patient lost $10 \mathrm{~kg}$ in weight. Levels of bilirubin and alanine aminotransferase were elevated. However, no signs of GVHD in the skin were observed. The glucosamine treatment was withdrawn and signs and symptoms of GVHD were successfully resolved by the treatment of cyclosporine and steroids orally and rectally. The patient is now in remission and there is no sign of GVHD or related problems for more than 4 years without immunosuppression. No explanation could be found for the late occurrence of acute GVHD except the administration of glucosamine.

\section{Case 2}

At the time of DLI administration (Fig. 1), the patient exhibited a poor donor chimerism $(\mathrm{CD} 3+=60 \%)$. However, administration of one low dose of DLI $\left(0.43 \times 10^{6} 6 \mathrm{CD} 3+\right.$ cells $/ \mathrm{kg}$ ) followed by the treatment with glucosamine one

\section{Patient no. 2}

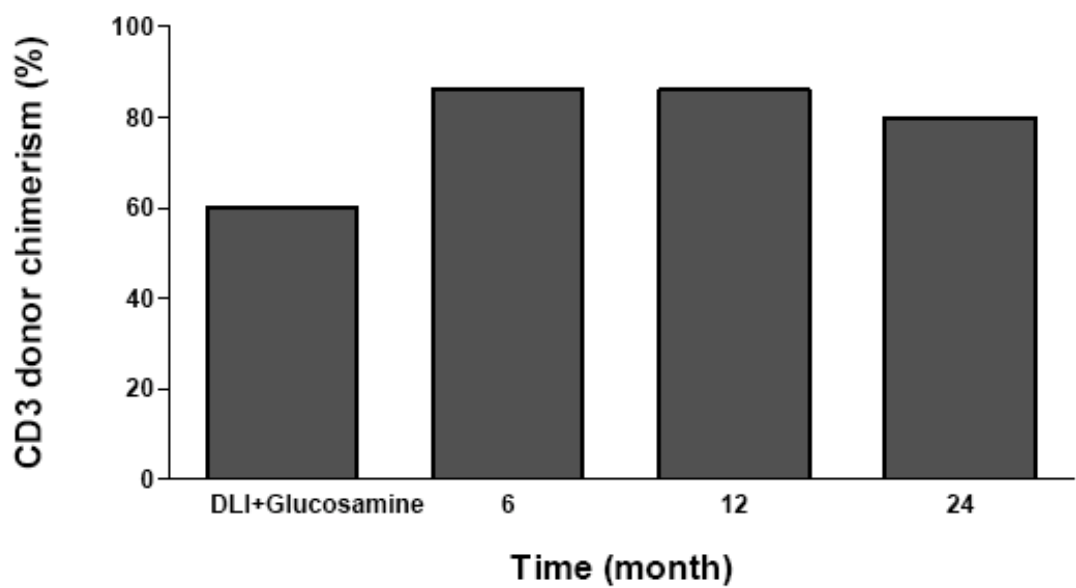

Fig. (1). CD3 T-lymphocyte chimerism changes in the recipient (patient no. 2). Donor T-lymphocyte population increased in peripheral blood Patient (no. 2) at 6, 12, and 24 months after one single dose of DLI in combination with the administration of glucosamine.

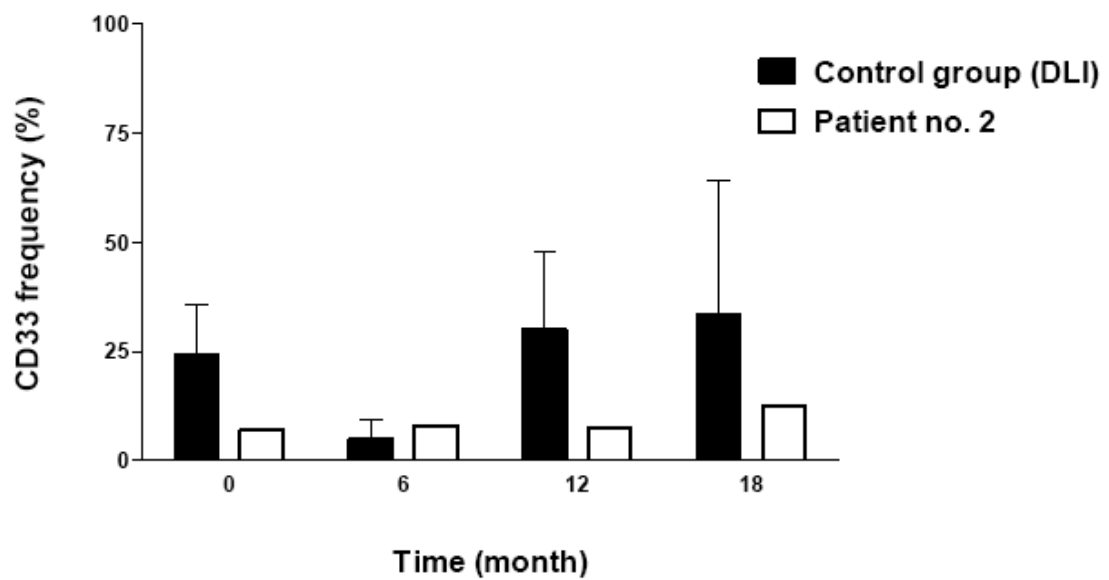

Fig. (2). Effect of glucosamine on relapse. Comparison of CD33+ levels (relapse marker) in the peripheral blood of the recipient (patient no. 2) and control group (patients that received several DLI at higher cell doses). Case no. 2 received fewer donor T cells $\left(0.043 \times 10^{7} \mathrm{CD} 3 / \mathrm{kg}\right)$ in combination with $1250 \mathrm{mg} /$ daily glucosamine $\left(\operatorname{Artrox}^{\circledR}\right)$. Still after 18 months she is in clinical remission whereas other patients regardless of receiving of 2.6 times DLI and mean of $1.78 \times 10^{7}$ donor CD3 cells have relapsed. 
moth later led to a significant increase in donor T-cell chimerism that Reach to its maximum level (82\%) after 6 months and remained at similar levels even after 2 years (Fig. 1). More importantly, upon DLI administration and further treatment with glucosamine, the frequency of recipient $\mathrm{CD} 33+$ cells remained at high level $(>10 \%)$ at all tested time points up to 2 years, indicating higher relapse risk (Fig. 2). In fact, four years after the treatment, the patient is clinically in complete remission with a minimal residual disease $(0.06 \%-0.5 \%)$ and there is no sign of relapse. These results indicate that treatment with glucosamine probably enhanced the efficiency of the DLI that in turn induced graft versus leukaemia reaction (GVL). In order to evaluate our hypothesis, we compared laboratory and clinical outcomes observed in this patient with the results found in the control group, who received DLI only. As shown in Fig. (2), in the absence of glucosamine, repeated administrations of DLI (Mean $=2.6$ times) at higher donor $\mathrm{CD} 3+$ cell concentrations $\left(\right.$ Mean $=1.78 \times 10^{7}$ ) was able to control the malignant cell (CD33+ cells) only for a short period of time ( 6 month), after which the frequency of these cells were substantially increased in most of the patients (Fig. 2). In line with this finding, all of these patients developed clinical sign of relapse and 5 out of 8 patients $(>60 \%)$ died within 20.6 months after HSCT.

\section{Glucosamine Administration Enhances the Lymphocyte Activity in Healthy Subjects}

Our findings that the treatment with glucosamine resulted in GVHD in first case led us to investigate if this drug may affect the immune system in healthy individuals. In order to answer this question, 7 healthy subjects were scheduled for the treatment with glucosamine in an identical fashion to drug-treated patients. At different intervals alloreactivity of the lymphocytes obtained from the treated individuals were determined employing MLR assay. As shown in Fig. (3), treatment with glucosamine induced a substantial increase in the allogeneic responses of lymphocytes, which initiated 2 weeks after treatment with glucosamine $(p=0.018)$ and reached a significant $(p=0.028)$ peak on week 4 (the termination time for the treatment). However, 8 weeks after the cessation of glucosamine (week 12), all the treated subjects exhibited an allogeneic response, which was similar to that found prior to the treatment with glucosamine (Fig. 3). This observation clearly implied that treatment with glucosamine enhances the alloreactivity of $\mathrm{T}$ lymph in vitro.

To further investigate if the treatment with glucosamine leads to the activation of $\mathrm{T}$ lymphocytes in vivo, we next measured the serum levels of sIL-2R, which is considered as a useful surrogate marker for $\mathrm{T}$ cell activation at different intervals [13]. As demonstrated in Fig. (4), after 2 weeks of treatment, glucosamine induced a significant increase in the serum levels of sIL-2R, which peaked on week 4 and thereafter, declined by the termination of the treatment.

\section{Effect of Glucosamine Administration on Inflammatory Cytokine Production in Healthy Individuals}

Since it is known that glucosamine possess anti-inflammatory effects $[9,10,12]$. We also monitored the serum levels of inflammatory cytokines, IFN- $\gamma$ and TNF- $\alpha$ in glucosamine treated healthy individuals employing chemoluminescence immunoassay. As shown in Fig. (5A), four weeks treatment with glucosamine resulted in a significant

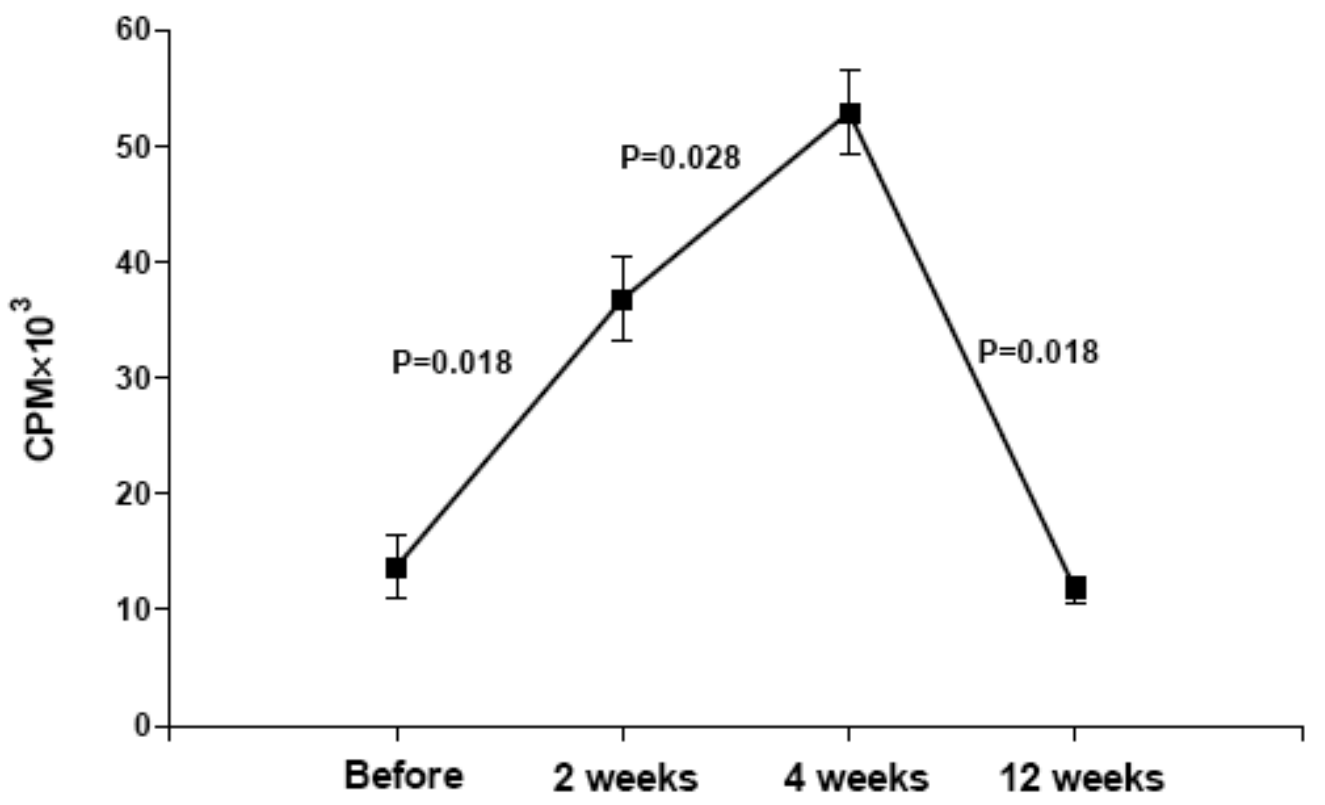

Time

Fig. (3). Effect of glucosamine on MLR in healthy volunteer. Seven healthy individuals administered glucosamine (1250mg/day) for four weeks. Lymphocytes from peripheral blood of these individual were used as responders to a pool of the 7 individuals that was used as stimulator. Mean values \pm SEM have shown at different time points before, 2, 4 (during the treatment) and 8 weeks after discontinuation of the glucosamine. $\mathrm{P}$ values indicate differences between each time point. (CPM= Counts/minute). 


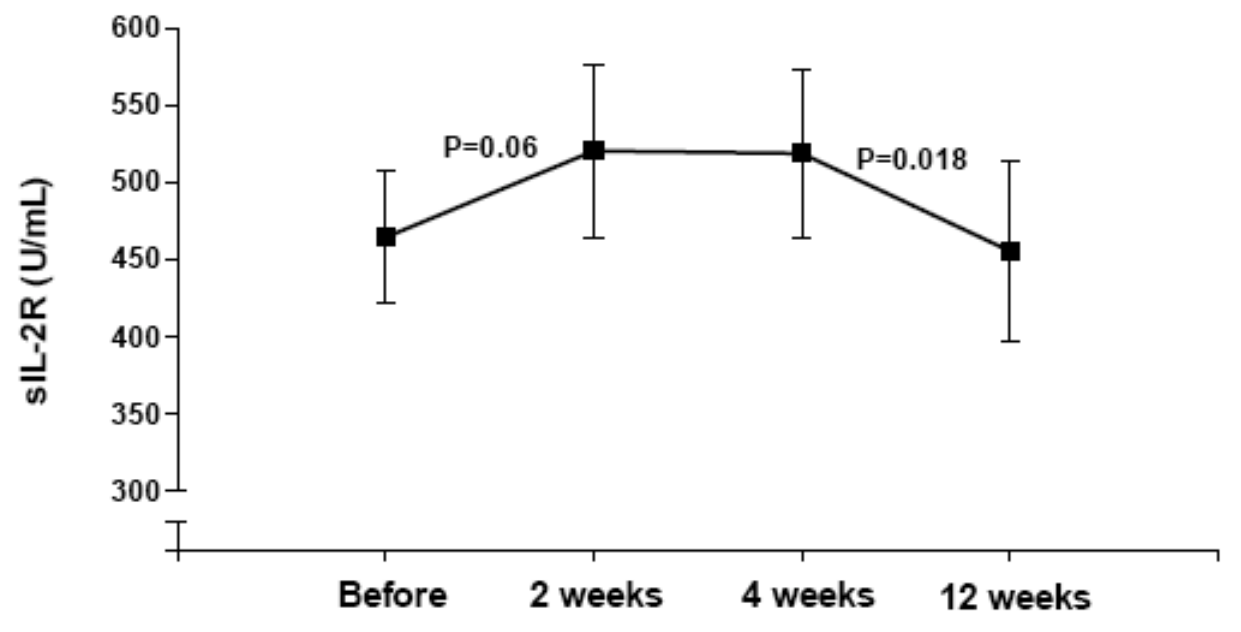

\section{Time}

Fig. (4). Effect of glucosamine on inflammatory cytokine secretion. Seven healthy individual received glucosamine (1250mg/day) for four weeks. Soluble Interleukin-2 receptor (sIL-2) was measured in the serum of individuals. Two and 4 weeks after taking glucosamine, serum levels of sIL2r increased from $464.3 \pm 43.4$ to $520.1 \pm 56.5(\mathrm{P}=0.06)$ and $518.5 \pm 54.8$, respectively. Eight weeks after discontinuation of glucosamine, sIL2r return to the normal level (455 \pm 58.8 ). Mean values \pm SEM are shown at different time points before, 2, 4 (during the treatment) and 8 weeks after discontinuation of the glucosamine. $\mathrm{P}$ values indicate differences between each time point.

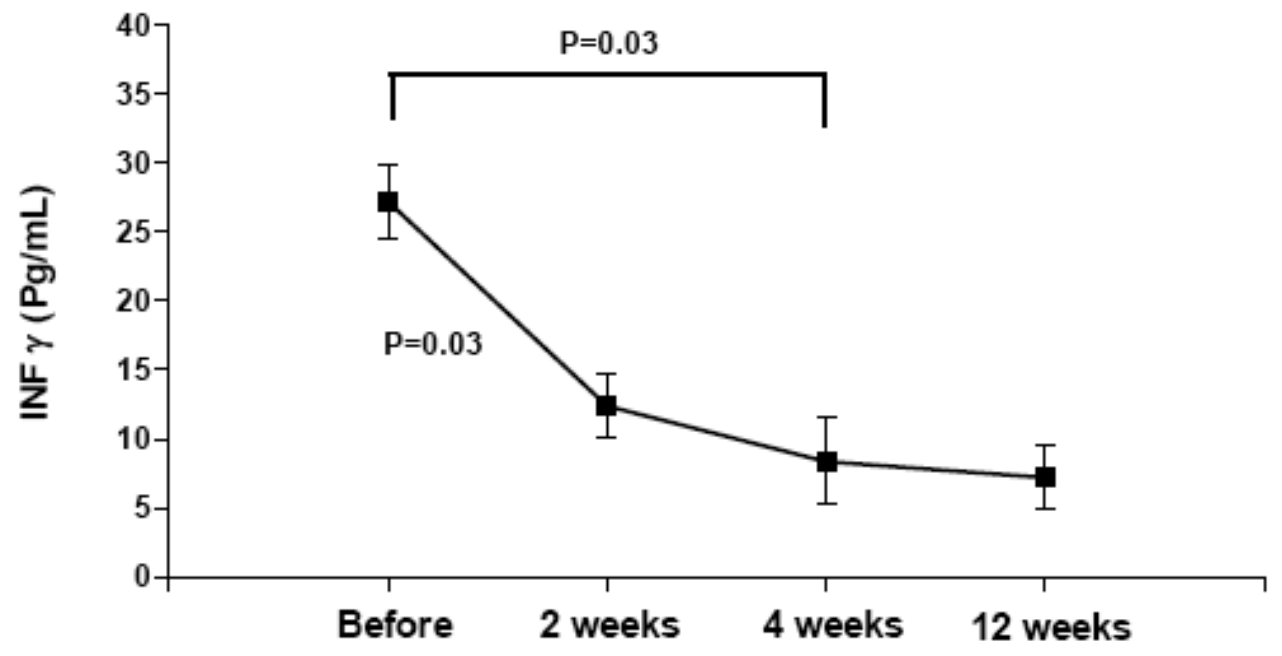

Time

Fig. (5A). Effect of Glucosamine on inflammatory cytokine secretion. Seven healthy individuals took received glucosamine (Artrox $\left.{ }^{\circledR}\right)$ at a dose of $1250 \mathrm{mg} /$ day for four weeks. Level of interferon $\gamma$ was measured in the serum of the individuals before and after administration of glucosamine. Two and 4 weeks after treatment with glucosamine serum levels of INF $\gamma$ decreased from 27.1 \pm 2.7 to $12.4 \pm 2.3$ and $8.3 \pm 3.1$, respectively comparing the levels found before administration. Mean values \pm SEM are shown at different time points before, 2, 4 (during the treatment) and 8 weeks after discontinuation of the glucosamine. $P$ values indicate differences between each time point.

decrease in the serum levels of IFN- $\gamma$, which initiated after 2 weeks and remained at the same reduced level even 8 weeks after the termination of the treatment. However, a nonsignificant decrease in the serum level of TNF- $\alpha$ was observed during the entire glucosamine treatment (Fig. 5B). Unfortunately, owing to a technical problem, we were not able to determine TNF- $\alpha$ level at 8 weeks after termination of glucosamine treatment.

\section{DISCUSSION}

It is well established that lymphocytes, particularly $\mathrm{T}$ lymphocytes contained in the allograft (either in the bone marrow or DLI) play an essential role in the outcome of alloHSCT [14]. That is, activation of donor $\mathrm{T}$ cells can mediate deleterious (e.g., GVHD) effects on the host [14], however, this effect may prevent graft rejection. Moreover, a beneficial effect can be obtained in form of graft versus 


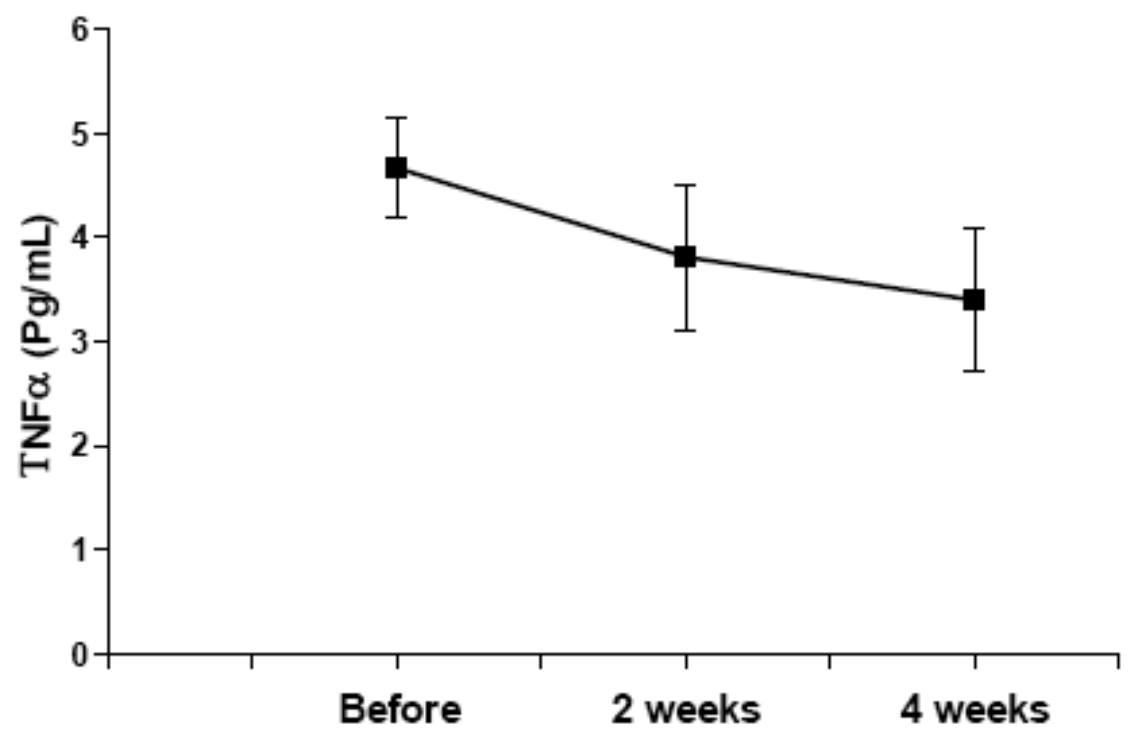

Time

Fig. (5B). Effect of Glucosamine on inflammatory cytokine secretion. Glucosamine (1250 mg/day) was given to seven healthy individuals for four weeks. Levels of TNF- $\alpha$ were measured in the serum of the subjects before and after administration of glucosamine. Two and 4 weeks after treatment with glucosamine, serum levels of TNF- $\alpha$ decreased from $4.8 \pm 0.5$ to $3.8 \pm 0.7$ and $3.4 \pm 0.7$, respectively comparing the value found before administration. Mean values \pm SEM have shown at different time points before, 2, 4 (during the treatment). Unfortunately serum sample was not enough to measure TNF- $\alpha$ levels at 8 weeks after discontinuation of the glucosamine.

leukemia effect. In other words, while in GVHD, there is a demand for the prevention or at least minimization of donor $\mathrm{T}$ cell activation, in GVL maximal or enhanced activation of donor $\mathrm{T}$ cell is required. Thus, this issue should be considered further when medications, especially OTC (over the counter) medicines, are used by and/or prescribed to HSCT patients.

In the present study, we observed that a HSCT patient in $\mathrm{CR}$ with a full donor chimerism when treated with an OTC drug, glucosamine, for a three weeks period, suddenly developed an acute GVHD. On the other hand, another patient who exhibited clinical signs of relapse after HSCT and received a single low dose of DLI in combination with glucosamine showed a sustained complete remission with a minimal residual disease even after 4 years. In fact, such condition was not observed in any of the control HSCT patients who received only DLI even at high frequencies and higher cell doses. These results strongly suggest that administration of glucosamine in HSCT patients may alter the outcome of the HSCT toward either a life threatening or a life saving condition depending on the patient situation.

The exact underlying mechanism for the development of GVHD and GVL in glucosamine-treated HSCT patient remains to be elucidated. However, as discussed in above, since activated donor $\mathrm{T}$ lymphocytes are known to be the main mediators of both GVHD and GVL in these patients, as the most probable mechanism, we suggest that glucosamine induces the activation of $\mathrm{T}$ lymphocyte in vivo to produce GVHD or GVL in HSCT patient. Indeed, this suggestion is strongly supported by our findings that in healthy individuals treatment with glucosamine enhanced the capacity of lymphocytes for alloreactive proliferation and increased the serum levels of sIL-2R, which is a marker for in vivo activation of $\mathrm{T}$ lymphocytes [13]. Moreover, this suggestion is further supported by the results of recent experimental studies in which it has been shown that oral administration of glucosamine to mice for 20 days significantly enhanced the specific antibody responses against the foreign antigen, sheep red blood cells (SRBC), increased the relative organ weight of spleen and thymus tissue, and promoted the delayed-type hypersensitivity (DTH) against SRBC [15]. Moreover, it has been demonstrated that in vitro, treatment with glucosamine exerts mitogenic effects and induces lymphocyte proliferation in the mouse lymphocytes [15].

In addition to the immunostimulatory effects of glucosamine found in the present study and as reported by Yan Y, et al. 2007, several other studies have shown that glucosamine can also exert anti-tumor activity [16-18]. For instance, Quastel et al. have shown that treatment with Dglucosamine is able to inhibit tumor growth in an experimental model of sarcoma [19]. Bekesi et al. have also reported that treatment with glucosamine exerted inhibitory effects on the growth of tumors in laboratory animals and that the treated animals were resistant to the implantation of second tumor cells [20]. Thus, regarding the anti-tumor activity of glucosamine, and our finding that the administration of glucosamine resulted in the development of GVL, it is likely that in this particular case, both immunostimulatory as well as anti tumor effects of glucosamie contribute to the development of GVL in this patient. Obviously, this likelihood is of high importance and is going to be addressed in our future studies.

As mentioned earlier in this study, glucosamine is well recognized as an anti inflammatory drug $[11,21]$. Although 
the exact underlying mechanism by which this drug induces anti inflammatory effects are not fully understood, the results from several studies suggests that glucosamine by acting on the innate immune cells particularly macrophages down regulates the expression of nuclear factor kappa B (NF-kB) and mitogen-activated protein kinases (MAPK), the key transcription factors that are required for the activation of inflammatory genes (e.g., TNF- $\alpha$, IFN- $\gamma$ and IL-1 $\beta$ ) [22-24]. In line with these studies we also found that administration of glucosamine reduced the serum levels of inflammatory cytokines, INF- $\gamma$ and TNF- $\alpha$ in healthy individuals. Thus, it is possible that treatment with glucosamine activates the cells of the adaptive branch of the immune system, but suppresses the activity of innate immune cells. Obviously, further studies are required to verify this possibility.

In conclusion: in the present study we showed for the first time that treatment with glucoseamine can exert immunostimulatory effects by activating $\mathrm{T}$ lymphocytes in healthy individuals and it may alter the outcome of bone marrow transplantation toward the development of either GVHD or GVL depending on the condition of the patient. Thus, considerable cautions should be made in the use of this OTC drug in bone marrow transplanted patients.

\section{ACKNOWLEDGEMENT}

The authors would like to express their gratitude to the Swedish Cancer Foundation (Cancer Fonden) and to the Swedish Children Cancer Society (Barncancer Fonden) for their support.

\section{REFERENCES}

[1] Storb R. Allogeneic hematopoietic stem cell transplantation-yesterday, today, and tomorrow. Exp Hematol 2003; 31(1): 1-10.

[2] Porter DL, Antin JH. The graft-versus-leukemia effects of allogeneic cell therapy. Annu Rev Med 1999; 50: 369-86.

[3] Champlin R, Giralt S, Gajewski J. T cells, graft-versus-host disease and graft-versus-leukemia: innovative approaches for blood and marrow transplantation. Acta Haematol 1996; 95(3-4): 157-63.

[4] Bassleer C, Rovati L, Franchimont P. Stimulation of proteoglycan production by glucosamine sulfate in chondrocytes isolated from human osteoarthritic articular cartilage in vitro. Osteoarthr Cartil 1998; 6(6): 427-34.

[5] Dodge GR, Jimenez SA. Glucosamine sulfate modulates the levels of aggrecan and matrix metalloproteinase- 3 synthesized by cultured human osteoarthritis articular chondrocytes. Osteoarthr Cartil 2003; 11(6): 424-32.

[6] Chan PS, Caron JP, Orth MW. Effect of glucosamine and chondroitin sulfate on regulation of gene expression of proteolytic enzymes and their inhibitors in interleukin-1-challenged bovine articular cartilage explants. Am J Vet Res 2005; 66(11): 1870-6.

[7] Reginster JY, Bruyere O, Neuprez A. Current role of glucosamine in the treatment of osteoarthritis. Rheumatology 2007; 46(5): 7315.

[8] Wilkens P. Effect of glucosamine on pain-related disability in patients with chronic low back pain and degenerative lumbar osteoarthritis: a randomized controlled trial. JAMA 2010; 304(1): 45-52.

[9] Shikhman AR. N-acetylglucosamine prevents IL-1 beta-mediated activation of human chondrocytes. J Immunol 2001; 166(8): 515560.

[10] Gouze JN. Glucosamine modulates IL-1-induced activation of rat chondrocytes at a receptor level, and by inhibiting the NF-kappa B pathway. FEBS Lett 2002; 510(3): 166-70.

[11] Forchhammer L. Immunobiological effects of glucosamine in vitro. Scand J Immunol 2003; 58(4): 404-11.

[12] Zhang GX. Glucosamine abrogates the acute phase of experimental autoimmune encephalomyelitis by induction of Th2 response. J Immunol 2005; 175(11): 7202-8.

[13] Mathias C. Soluble interleukin-2 receptor concentration as a biochemical indicator for acute graft-versus-host disease after allogeneic bone marrow transplantation. J Hematother Stem Cell Res 2000; 9(3): 393-400.

[14] Sprangers B. Can graft-versus-leukemia reactivity be dissociated from graft-versus-host disease? Front Biosci 2007; 12: 4568-94.

[15] Yan Y. The antioxidative and immunostimulating properties of Dglucosamine. Int Immunopharmacol 2007; 7(1): 29-35.

[16] Bosmann HB. Inhibition of protein, glycoprotein, ribonucleic acid and deoxyribonucleic acid synthesis by D-glucosamine and other sugars in mouse leukemic cells L5178Y and selective inhibition in SV-3T3 compared with 3T3 cells. Biochim Biophys Acta 1971; 240(1): 74-93.

[17] Friedman SJ. The inhibition of thymidine metabolism in tumor cells treated with D-glucosamine. Cancer Res 1977; 37(4): 1141-6.

[18] Bekesi JG, Bekesi E, Winzler RJ. Inhibitory effect of Dglucosamine and other sugars on the biosynthesis of protein, ribonucleic acid, and deoxyribonucleic acid in normal and neoplastic tissues. J Biol Chem 1969; 244(14): 3766-72.

[19] Quastel JH, Cantero A. Inhibition of tumour growth by Dglucosamine. Nature 1953; 171(4345): 252-4.

[20] Bekesi JG, Winzler RJ. Inhibitory effects of D-glucosamine on the growth of Walker 256 carcinosarcoma and on protein, RNA, and DNA synthesis. Cancer Res 1970; 30(12): 2905-12.

[21] Ma L. Immunosuppressive effects of glucosamine. J Biol Chem 2002; 277(42): 39343-9.

[22] Largo R. Glucosamine inhibits IL-1beta-induced NFkappaB activation in human osteoarthritic chondrocytes. Osteoarthr Cartil 2003; 11(4): 290-8.

[23] Mendis E. Suppression of cytokine production in lipopolysaccharide-stimulated mouse macrophages by novel cationic glucosamine derivative involves down-regulation of NF-kappaB and MAPK expressions. Bioorg Med Chem 2008; 16(18): 8390-6.

[24] Rajapakse N. Inhibition of inducible nitric oxide synthase and cyclooxygenase- 2 in lipopolysaccharide-stimulated RAW264.7 cells by carboxybutyrylated glucosamine takes place via downregulation of mitogen-activated protein kinase-mediated nuclear factor-kappaB signaling. Immunology 2008; 123(3): 348-57. 